\title{
The dynamics analysis of automobile chassis for shelter
}

\author{
Xi Duan ${ }^{1, \mathrm{a}}$, Youyong Ding ${ }^{1, \mathrm{~b}}$, Yangyang $\mathrm{Hu}^{1, \mathrm{c}}$ \\ ${ }^{1}$ No. 1,Yong Zhi Road, Qinhuai District, Nanjing, Jiangsu Province, China \\ aduanxi1985@163.com, ${ }^{\text {b703371656@qq.com, }{ }^{c} x u z h o u h y y @ 163 . c o m ~}$
}

Key words: Automobile chassis; Crosse the obstacle; Dynamics analysis

Abstract: The shelter generally carried by automobile chassis. Therefore it needs corresponding automobile chassis to adapt to different road conditions. The three types of common obstacle are ravine, slope and step. When crossing the three types of obstacles, the dynamics analysis of automobile chassis can be used for checking whether the automobile chassis can meet the requirements or not. It also can be used for some key parameters checking before the secondary refit of the automobile chassis.

\section{Obstacle types}

The shelter generally carried by automobile chassis (Fig. 1). In the face of various complicated road conditions, we need to choose appropriate automobile chassis with corresponding obstacle capability, or refit the automobile chassis according to actual needs, to fit the needs of shelter.

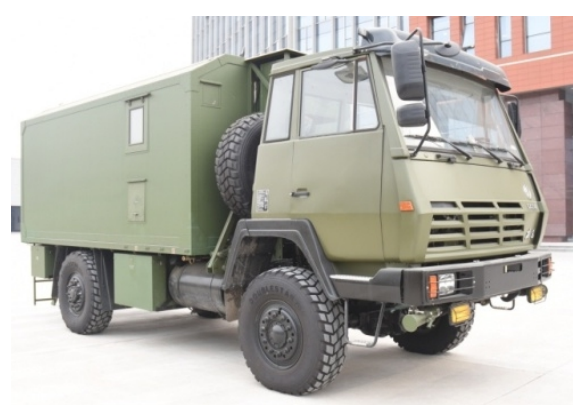

Figure 1 A shelter on automobile chassis

In order to assess the capability of automobile chassis to cross obstacle, all kinds of obstacles can be abstracted into three types, which are gully, slope and stair (Fig. 2, Fig. 3). Dynamic analysis in automobile chassis through the three barriers not only be related to the maximum loading weight, but also to the power of the engine, car driving form, transmission mechanism design and ground environmental factors ${ }^{[1]}$.

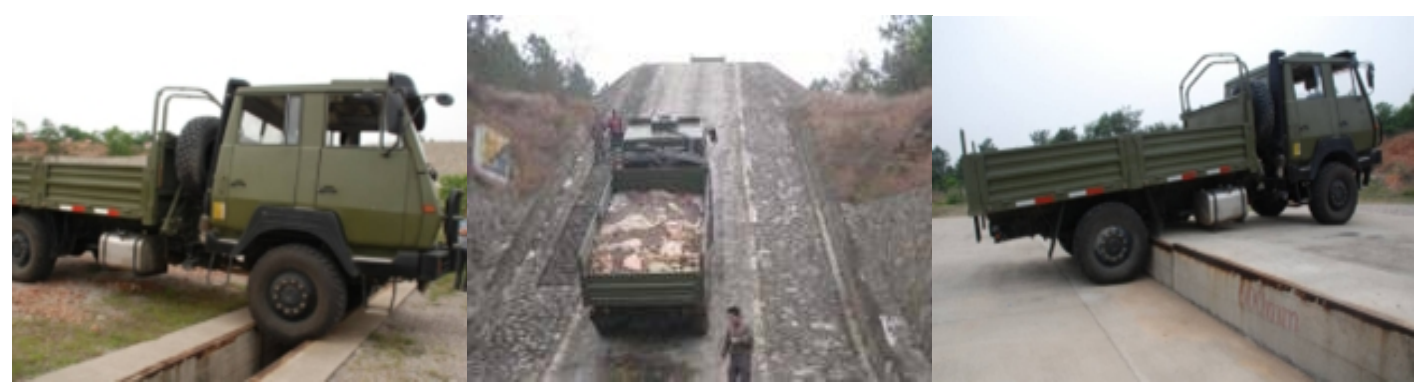

Figure 2 The obstacle test of a vehicle 


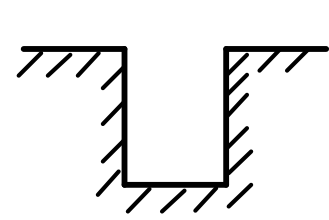

A. Gll ly

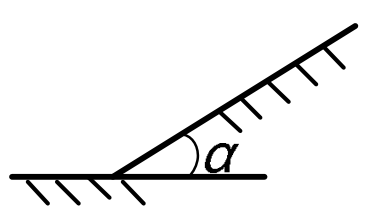

B. Sl ope

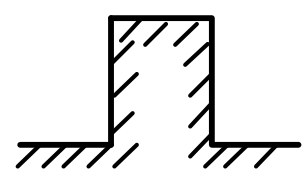

C. Step

Figure 3 Schematic diagrams of three types of obstacles

During the layout design of shelter, We usually make the center of gravity of the vehicle close to the geometric center, so in this paper, the dynamic analysis of crossing the three kinds of obstacles on the automobile chassis are assumed at the geometrical center, and once the tire slip over the gully or the stairs, means that it fails to cross the obstacle, so we assume that the tire only rolls ${ }^{[2]}$. Off-road vehicle chassis is generally $4 \times 4$ or $6 \times 6$ multi wheel drive, in order to facilitate the calculation, this article is set to be $4 \mathrm{WD}^{[3]}$.

\section{Analysis of three cases of obstacle}

Crossing gully and the force analysis. The case of the automobile chassis crossing the gully is shown in Fig. 4.

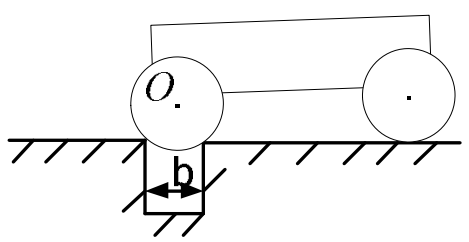

St age [

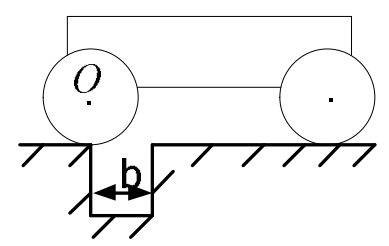

St age [

Figure 4 The situation of automobile chassis crossing the gully

As long as making the wheel center point $O$ crossing the point where the vertical edge of the gully, it is safe to cross the gully. In view of the limitations of wheeled vehicle chassis, in general, the width of the $b$ is less than the diameter of the wheel, otherwise the chassis will be stuck on the edge of the ravine, leading to chassis damage.

When the current tire just climbed out of the gully, only contact with the front edge of the ravine, and the back edge of the supporting force of friction is 0 , the force analysis is shown in Fig. 5

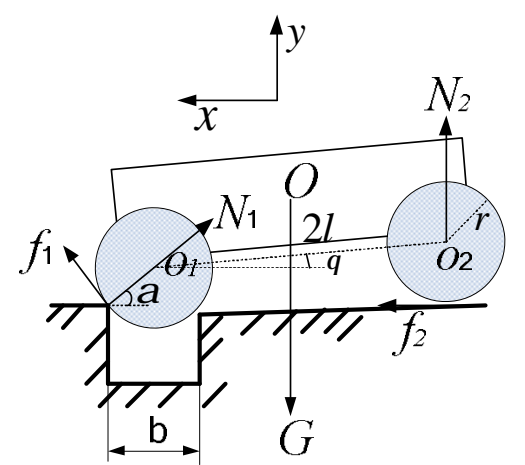

Figure 5 The force analysis of the automobile chassis when crossing gully

When the front tire of the automobile chassis can just climb out of the gully, the force balance equation is: 


$$
\left\{\begin{array}{l}
\sum F(x)=0, f_{1} \sqsubset \sin \alpha+f_{2}-N_{1} \cos \alpha=0 \\
\sum F(y)=0, f_{1} \sqsubset \cos \alpha+N_{1} \sin \alpha+N_{2}-G=0
\end{array}\right.
$$

Supplementary equation is:

$$
\left\{\begin{array}{l}
f_{1}=\mu N_{1} \\
f_{2}=\mu N_{2} \\
\alpha=\arccos \frac{b}{2 r}
\end{array}\right.
$$

Analysis methods are similar when the rear wheels fall into the gully. The equation can be used to calculate the relationship between the traction force and the gully width $b$. At the same time, the required engine power can be obtained according to the present shelter car chassis speed by Eq. 3 .

$$
P=F v
$$

Climbing slope and the force analysis. There is a limit to the climbing ability of the chassis, and the ability can be expressed by the ratio of the height $h$ to the horizontal distance $b$, and this value is also the tangent angle of slope, which is:

$$
\tan \alpha=\frac{h}{b}
$$

Generally, Some good off-road performance vehicle climbing capacity can be around 60\%, China Military Museum lobby has a China independently developed military off-road vehicle, its climbing capacity is $62 \%$, which can be converted into the equivalent of $32^{\circ}$. Automobile chassis generally requires climbing capacity no less than $30^{\circ}$.

When the car chassis on the slope is in uniform motion, the force analysis is shown in Fig. 6, which $F$ for traction, $f$ for rolling resistance.

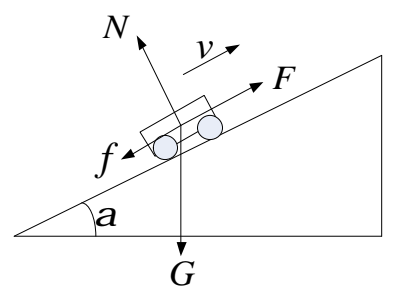

Figure 6 The force analysis of the automobile chassis when climbing slope The force balance equation is:

$$
\left\{\begin{array}{l}
F=f+G \sin \alpha \\
N=G \cos \alpha
\end{array}\right.
$$

Which $f=\lambda N$, and the traction can be derived: 


$$
F=G(\lambda \cos \alpha+\sin \alpha)
$$

It can be seen that the magnitude of the force required to climb the slope is related to the weight of the chassis, the slope of the slope and the coefficient of rolling resistance.

The Eq. 6 can be seen as a function of $F$ on $\alpha$. According to mathematical knowledge, the Eq. 6 can be changed into:

$$
F=G \sqrt{1+\lambda^{2}} \sin (\theta+\alpha)
$$

Which $\tan \theta=\lambda, 0<\theta<\frac{\pi}{4}$ can be seen by $\lambda<1$, and normally $0<\alpha<\frac{\pi}{4}$, therefore the Eq. 7 is an increasing function in the interval $\left[0, \frac{\pi}{2}\right]$. With the increase of the slope, it needs more and more traction, in the case of a certain power, the speed must be reduced in order to ensure the traction.

Of course, the slope cannot be increased indefinitely, when reaching a critical point, the car chassis gravity along the maximum static friction force is greater than which the slope downward slope can provide, the car chassis tires began to slide on the slopes and down slowly ${ }^{[4]}$.

Climbing step and the force analysis. Firstly, we consider that the height of the step is less than the radius of the tire, as shown in Fig. 7:

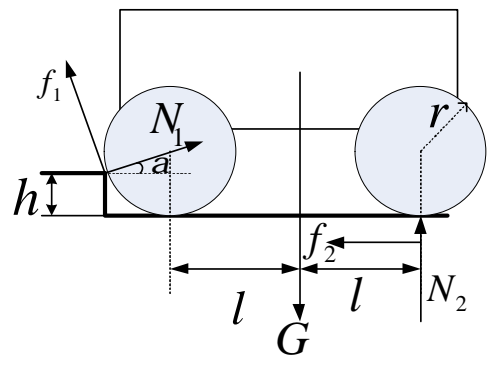

Figure 7 The force analysis of the automobile chassis when climbing step

We also assume that the front tire can climb stairs, when it is just off the ground, by force balance equation:

$$
\left\{\begin{array}{l}
f_{1} \cos \alpha+N_{1} \sin \alpha+N_{2}=G, f_{1}=\mu N_{1} \\
f_{1} \sin \alpha+f_{2}=N_{1} \cos \alpha, f_{2}=\mu N_{2} \\
\left(f_{1} \cos \alpha+N_{1} \sin \alpha\right)(l+r)=N_{2} l
\end{array}\right.
$$

Simplification equation can be obtained:

$$
2 \mu \tan \alpha=1-\mu^{2}\left(1+\frac{r}{l}\right)
$$

Which $\tan \alpha=\frac{r-h}{\sqrt{h(2 r-h)}}$, when $h$ is infinitely less than $r$ the steps tend to be flat, and the chassis can be analyzed according to the ground level. when ${ }_{h \geq r}$, The direction of $N$ becomes level, $\tan \alpha=0$, we can obtain that: 


$$
1+\frac{r}{l}=\frac{1}{\mu^{2}}
$$

Eq. 10 shows that in sufficient power, whether the chassis can climb stairs or not, depends on the tire radius, the wheelbase and the friction coefficient between the tire and the wheelbase.

Actually because $r<l$, we can find that $\mu>0.707$, And the closer the coefficient is to 0.7 , the closer the two wheels are, but the layout is not reasonable. In order to ensure the loading ability, generally the off-road chassis wheelbase is 2.5 times above than the diameter of the tire, through calculation, and off-road tires in dry cement or asphalt pavement, can obtain the maximum friction coefficient from 0.7 to 0.8 . Therefore, when the height of the step is greater than or equal to the radius of the tire, generally the off-road vehicle cannot cross the step.

If the tire diameters, the wheelbase of the automobile chassis have been known, the friction coefficient can be calculated by the obstacle height of automobile chassis and engine power required. If the height of step has been known, plug the other values can check out that whether the chassis can successfully assess obstacle or not ${ }^{[5]}$.

\section{Conclusions}

When the automobile chassis is provided with shelter and ancillary equipment, vehicle weight and the center of the whole system will change a lot, so according to specific conditions of square cabin and automobile chassis overall dynamics analysis, to check whether the chassis power, wheelbase and drive form can meet the requirements of the corresponding obstacle or not. At the same time, it also has certain reference significance in this paper when doing some modification calculation and analysis of obstacle surmounting before modifying the automobile chassis.

\section{Reference}

[1] Hou Jinzhong. A brief analysis of cross-country performance of military off-road vehicles. Heavy duty truck. 1997(2), p.8-10.

[2] Guo Zhanjun, Zhang Weigong, Pang Han. Typical terrain modeling and Simulation of off-road vehicle. Automobile design. 2008(11), p.31-33.

[3] Liu Shikai. Structural principle of automobile transmission system. China Communications Press. 1996, p.9

[4] Yang Hangtong, Analysis of carrier vehicle grade ability , Mechanical Electrical Engineering , 1998(1),p.49-50.

[5] Lan Fengchong, Chen Jiqing, Wang yu. Calculation and test of vertical obstacle capability of automobile, Automotive Engineering, 1997, 19(2), p.116-120. 